\title{
A new method to diagnose the contribution of anthropogenic activities to temperature: temperature tagging
}

\author{
V. Grewe \\ Deutsches Zentrum für Luft- und Raumfahrt, Institut für Physik der Atmosphäre, Oberpfaffenhofen, Germany \\ Correspondence to: V. Grewe (volker.grewe@dlr.de)
}

Received: 29 August 2012 - Published in Geosci. Model Dev. Discuss.: 12 October 2012

Revised: 3 March 2013 - Accepted: 12 March 2013 - Published: 26 March 2013

\begin{abstract}
This study presents a new methodology, called temperature tagging. It keeps track of the contributions of individual processes to temperature within a climate model simulation. As a first step and as a test bed, a simple box climate model is regarded. The model consists of an atmosphere, which absorbs and emits radiation, and of a surface, which reflects, absorbs and emits radiation. The tagging methodology is used to investigate the impact of the atmosphere on surface temperature. Four processes are investigated in more detail and their contribution to the surface temperature quantified: (i) shortwave influx and shortwave atmospheric absorption ("sw"), (ii) longwave atmospheric absorption due to non- $\mathrm{CO}_{2}$ greenhouse gases ("nC"), (iii) due to a base case $\mathrm{CO}_{2}$ concentration ("bC"), and (iv) due to an enhanced $\mathrm{CO}_{2}$ concentration ("eC"). The differential equation for the temperature in the box climate model is decomposed into four equations for the tagged temperatures. This method is applied to investigate the contribution of longwave absorption to the surface temperature (greenhouse effect), which is calculated to be $68 \mathrm{~K}$. This estimate contrasts an alternative calculation of the greenhouse effect of slightly more than $30 \mathrm{~K}$ based on the difference of the surface temperature with and without an atmosphere. The difference of the two estimates is due to a shortwave cooling effect and a reduced contribution of the shortwave to the total downward flux: the shortwave absorption of the atmosphere results in a reduced net shortwave flux at the surface of $192 \mathrm{~W} \mathrm{~m}^{-2}$, leading to a cooling of the surface by $14 \mathrm{~K}$. Introducing an atmosphere results in a downward longwave flux at the surface due to atmospheric absorption of $189 \mathrm{~W} \mathrm{~m}^{-2}$, which roughly equals the net shortwave flux of $192 \mathrm{~W} \mathrm{~m}^{-2}$. This longwave flux is a result of both the radiation due to atmospheric temperatures and its longwave absorption. Hence the
\end{abstract}

longwave absorption roughly accounts for $91 \mathrm{~W} \mathrm{~m}^{-2}$ out of a total of $381 \mathrm{~W} \mathrm{~m}^{-2}$ (roughly $25 \%$ ) and therefore accounts for a temperature change of $68 \mathrm{~K}$. In a second experiment, the $\mathrm{CO}_{2}$ concentration is doubled, which leads to an increase in surface temperature of $1.2 \mathrm{~K}$, resulting from a temperature increase due to $\mathrm{CO}_{2}$ of $1.9 \mathrm{~K}$, due to non- $\mathrm{CO}_{2}$ greenhouse gases of $0.6 \mathrm{~K}$ and a cooling of $1.3 \mathrm{~K}$ due to a reduced importance of the solar heating for the surface and atmospheric temperatures. These two experiments show the feasibility of temperature tagging and its potential as a diagnostic for climate simulations.

\section{Introduction}

Whenever an extreme weather event happens, like the Russian heat wave in August 2010, the question is posed whether this heat wave is a consequence of climate change. Such questions have been addressed in various ways. Hansen et al. (2012) investigated the likelihood that such an event happened in a world without climate change and concluded that this event is a consequence of global warming since its "likelihood in the absence of global warming was exceedingly small." On the other hand, Dole et al. (2011) showed that the Russian heat wave was primarily a consequence of a blocking event, a specific weather pattern not unusual for Russia. In ensemble simulations they showed that this blocking was not primarily caused by either greenhouse gases or sea surface temperatures and concluded that this specific weather event was solely caused by internal variability and that no anthropogenic influence was detected. The attribution techniques are different in both studies. However, what both studies have in common is that they compare on a statistical 
basis a world with climate change to a world without climate change (Hansen et al., 2012) or to a world with changed climate forcings (Dole et al., 2011). Here, a new methodology is introduced, which is not based on such statistical approaches, but deterministically attributes contributions of individual processes to the temperature: a temperature tagging methodology. Hence, with this method it is possible to answer the following question: how much of an observed heat wave is actually contributed by anthropogenic greenhouse gas emissions? Note that tagging is not addressing the question of whether a heat wave would also have happened in a world without climate warming.

This methodology builds on tagging techniques developed in atmospheric chemistry (Grewe et al., 2010). These techniques enable fully decomposing a non-linear system and attributing chemical concentrations to emissions of gases. The principles of this technique are applied in this paper to the contribution of atmospheric absorption of radiation to the temperature in a very simple box climate model. It shows the feasibility and the potential of such a technique and should be seen as a first step towards a full temperature tagging method in climate or weather prediction models, with which the question of the contribution of anthropogenic greenhouse gas emissions to the temperature during an individual weather event can be answered.

The temperature tagging method in a simple climate box model is applied to investigate the contribution of atmospheric longwave absorption (greenhouse effect) to surface temperature and the temperature contribution from a doubling of the $\mathrm{CO}_{2}$ concentration.

In the next section, basic definitions, like tagging categories, are given (Sect. 2.1). The simple climate box model is introduced in Sect. 2.2, and the temperature tagging methodology is presented in Sect. 2.3, with the experimental set-up presented in Sect. 2.4. Results are analysed in Sect. 3, first on the contribution of the greenhouse effect (GHE) on the surface temperature (Sect. 3.1) and second the contribution of a doubling of the $\mathrm{CO}_{2}$ concentration to the surface temperature (Sect. 3.2). A very brief outlook on how this methodology can be applied to a comprehensive climate model is given in Sect. 4 , followed by conclusions.

\section{Methodology}

In this section a very simple climate box model is presented (Sect. 2.2), as it can be found in many meteorology text books (e.g. Andrews, 2010), used to test the idea of a temperature tagging (Sect. 2.3). This is a method to track changes in a model simulation induced by any regarded process, e.g. temperature changes induced by greenhouse gases. Depending on the scientific question, there might be many possibilities to define temperature categories. Therefore Sect. 2.1 starts with the definition of the scientific question and the associated tagging categories.
Table 1. Overview on tagging categories.

\begin{tabular}{lcccc}
\hline Description & Category & \multicolumn{2}{c}{ Tagged } & \multicolumn{2}{c}{ Absorption } \\
& & temperature & sw & lw \\
\hline shortwave contribution & "sw" & $T^{\mathrm{sw}}$ & $a_{\mathrm{s}}$ & 0 \\
non- $\mathrm{CO}_{2} \mathrm{GHE}$ & "nC" & $T^{\mathrm{nC}}$ & 0 & $a^{\mathrm{nC}}$ \\
base $\mathrm{CO}_{2} \mathrm{GHE}$ & "bC" & $T^{\mathrm{bC}}$ & 0 & $a^{\mathrm{bC}}$ \\
GHE due to enhanced $\mathrm{CO}_{2}$ & "eC" & $T^{\mathrm{eC}}$ & 0 & $a^{\mathrm{eC}}$ \\
\hline
\end{tabular}

For this kind of tagging two aspects are important to be mentioned. Tagging has to be complete but not necessarily a material quantity. Completeness means that categories have to be defined in a way that the regarded physical quantity (here temperature) is totally split into individual parts without any remaining parts. Another way of looking at it is that the quantity is split into contributions and the sum of the contributions is again $100 \%$. Note that individual contributions might also be negative, e.g. for a cooling process. Hence, the tagged temperatures cannot be seen as physical temperatures, but as contributions to the temperature.

The second aspect is that tagging has mainly been used for chemical simulations, i.e. putting a tag to a material quantity, like ozone concentrations. This facilitates the tagging concept, but is physically or mathematically not necessary. Tags can be viewed as contributions of a process to any regarded quantity (Grewe, 2013).

This tagging concept leads to a two-step approach within one simulated model timestep. First the normal physical system is solved (here described in Sect. 2.2), and afterwards a second set of differential equations is solved for the tagged quantities (Sect. 2.3).

\subsection{Definition of tagging categories}

The underlying scientific question, addressed here, is how large the contribution is of the absorption of longwave radiation caused by greenhouse gases to the surface temperature. Therefore, four different effects are of interest, which affect temperatures (Table 1). The first is the effect of the shortwave solar input and shortwave atmospheric absorption, tagged with the name "sw". The transmitted solar radiation heats the surface and contributes thereby to the emission of longwave radiation, which in turn affects the atmospheric temperature by longwave absorption and feeds back to the surface temperature.

The other effects are related to the greenhouse effect, i.e. the longwave atmospheric absorption of radiation due to greenhouse gases and again their effects on surface and atmospheric temperatures. In order to study individual greenhouse gases, three groups are defined: all non- $\mathrm{CO}_{2}$ greenhouse gases, called " $\mathrm{nC}$ "; a base $\mathrm{CO}_{2}$ concentration (here: $360 \mathrm{ppm}$ ), called "bC"; and an enhanced $\mathrm{CO}_{2}$ concentration ("eC") of 360 ppm, representing a $\mathrm{CO}_{2}$ doubling. (Latter concentration is set to $0 \mathrm{ppm}$, if not used.) 


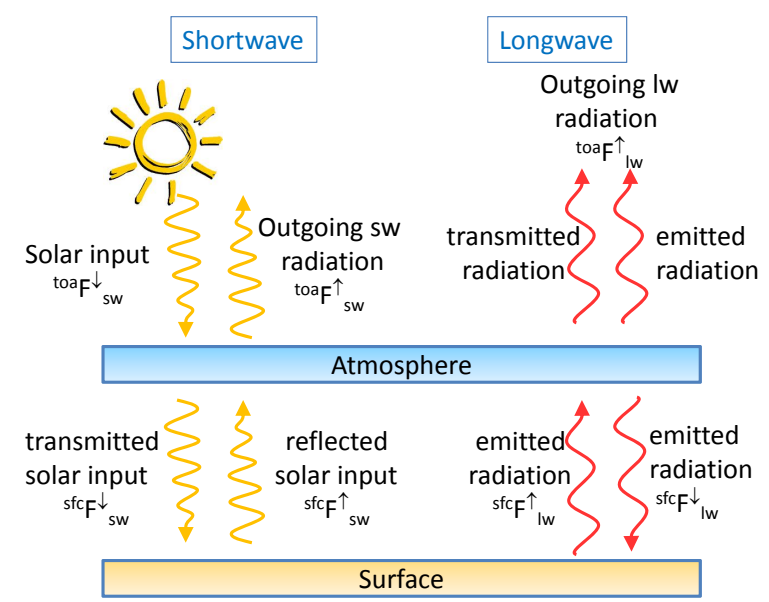

Fig. 1. Sketch of a simple climate box model.

This leads to four tagging categories: "sw", "nC", "bC", "eC" (see also Table 1), which are physically defined in the following two sections.

\subsection{A very simple climate model as a test bed}

The basic concept of this climate box model is presented in Fig. 1. It consists of two domains. The first domain is the atmosphere with a temperature $T_{\mathrm{a}}$. The atmosphere absorbs radiation in the shortwave $\left(a_{\mathrm{s}}\right)$ and longwave $\left(a_{1}\right)$. The second domain is the surface, characterised by the temperature $T_{\mathrm{S}}$ and the albedo $(A)$. Table 2 gives an overview on the chosen parameters.

At the top of the atmosphere (toa), the downward shortwave flux equals the solar radiation:

${ }^{\text {toa }} F_{\mathrm{sw}}^{\downarrow}=S=342 \mathrm{~W} \mathrm{~m}^{-2}$.

The atmosphere absorbs shortwave radiation, and with the assumption of no reflection or scattering the downward flux at the surface (sfc) is

${ }^{\mathrm{sfc}} F_{\mathrm{sw}}^{\downarrow}=\left(1-a_{\mathrm{s}}\right)^{\text {toa }} F_{\mathrm{sw}}^{\downarrow}$.

A part of this radiation is reflected, and the resulting upward directed flux is partly absorbed by the atmosphere before leaving the atmosphere:

${ }^{\text {sfc }} F_{\mathrm{sw}}^{\uparrow}=A^{\mathrm{sfc}} F_{\mathrm{sw}}^{\downarrow}$

${ }^{\text {toa }} F_{\mathrm{sw}}^{\uparrow}=\left(1-a_{\mathrm{s}}\right)^{\mathrm{sfc}} F_{\mathrm{sw}}^{\uparrow}$.

The temperature of the surface leads to an upward directed longwave radiation according to the Stefan-Boltzmann law:

${ }^{\mathrm{sfc}} F_{\mathrm{lw}}^{\uparrow}=\epsilon \sigma T_{\mathrm{s}}^{4}$.

By using Kirchhoff's law, which states that the longwave emission equals the absorption, the atmosphere radiates with
Table 2. Overview on parameters for the climate box model $\mathrm{lw}=$ longwave; $\mathrm{sw}=$ shortwave.

\begin{tabular}{|c|c|c|c|}
\hline Parameter & Description & Value & Unit \\
\hline$S$ & Solar input $^{1}$ & 342 & $\mathrm{Wm}^{-2}$ \\
\hline$a_{1}$ & lw absorption ${ }^{2}$ & variable & - \\
\hline$a_{\mathrm{S}}$ & sw absorption ${ }^{3}$ & 0.2 & - \\
\hline$A$ & Albedo 1 & 0.3 & - \\
\hline$\sigma$ & Stefan-Boltzmann constant & $5.67 \times 10^{-8}$ & $\mathrm{Wm}^{-2} \mathrm{~K}^{-4}$ \\
\hline$\epsilon$ & Emissivity $^{1}$ & 1 & - \\
\hline$c_{p}^{\mathrm{air}}$ & Specific heat capacity (air) ${ }^{4}$ & 1004 & $\mathrm{~J} \mathrm{~kg}^{-1} \mathrm{~K}^{-1}$ \\
\hline$c_{p}^{p s c}$ & Specific heat capacity $(\mathrm{sfc})^{5}$ & 3025 & $\mathrm{~J} \mathrm{~kg}^{-1} \mathrm{~K}^{-1}$ \\
\hline$\rho^{\text {air }}$ & Mean atmospheric density 6 & 0.5 & $\mathrm{~kg} \mathrm{~m}^{-3}$ \\
\hline$\rho^{\mathrm{sfc}}$ & Mean surface density ${ }^{6}$ & 1000 & $\mathrm{~kg} \mathrm{~m}^{-3}$ \\
\hline$Z^{\text {air }}$ & Atmospheric height $^{6}$ & 10000 & $\mathrm{~m}$ \\
\hline$Z^{\mathrm{sfc}}$ & Surface layer depth 6 & 1000 & $\mathrm{~m}$ \\
\hline$T_{\mathrm{a}}$ & Atmospheric temperature & variable & $\mathrm{K}$ \\
\hline & Surface temperature & variable & $\mathrm{K}$ \\
\hline${ }^{\text {toa }} F_{\mathrm{sw}}^{\uparrow}$ & $\begin{array}{l}\text { Top of the atmosphere } \\
\text { upward shortwave radiation } \\
\text { (also for sfc.; downward; lw) }\end{array}$ & variable & $\mathrm{Wm}^{-2}$ \\
\hline
\end{tabular}

${ }^{1}$ Taken from Andrews (2010). ${ }^{2}$ Absorption is parameterised, depending on the greenhouse gas concentrations. It is chosen as 0.77 for a current situation with 360 ppmv $\mathrm{CO}_{2} \cdot{ }^{3}$ Taken from Kiehl and Trenberth (1997). ${ }^{4}$ Taken from Hilsenrath et al. (1955). ${ }^{5}$ The surface heat capacity $c_{p}^{\mathrm{sfc}}$ is a combination of the heat capacities of $2 / 3$ of water (4180 $\mathrm{Jg}^{-1} \mathrm{~K}^{-1}$, Osborne et al., 1939) and $1 / 3$ of soils $\left(720 \mathrm{~J} \mathrm{~kg}^{-1} \mathrm{~K}^{-1}\right.$ out of a range of $625-1045 \mathrm{~J} / \mathrm{kg} / \mathrm{K}$; Bowers and Hanks , 1962).

${ }^{6}$ Scale assumption for this work.

a longwave flux $a_{1} \sigma T_{\mathrm{a}}^{4}$, which equals the downward longwave radiation at the surface:

${ }^{\text {sfc }} F_{\mathrm{lw}}^{\downarrow}=a_{1} \sigma T_{\mathrm{a}}^{4}$.

This contributes to the longwave outgoing radiation in addition to the transmittance of the upward directed longwave surface flux:

${ }^{\text {toa }} F_{\mathrm{lw}}^{\uparrow}=\left(1-a_{1}\right){ }^{\mathrm{sfc}} F_{\mathrm{lw}}^{\uparrow}+a_{1} \sigma T_{\mathrm{a}}^{4}$.

In steady state the fluxes are balanced; i.e. at top of the atmosphere (toa) and surface (sfc), both net fluxes ( ${ }^{\text {toa }} F_{\text {net }}$ and $\left.{ }^{\mathrm{sfc}} F_{\text {net }}\right)$ are zero. Hence the temperatures for the surface and the atmosphere can be deduced directly from

$$
\begin{aligned}
{ }^{\text {toa }} F_{\text {net }} & =-{ }^{\text {toa }} F_{\mathrm{lw}}^{\uparrow}+{ }^{\text {toa }} F_{\mathrm{sw}}^{\downarrow}-{ }^{\text {toa }} F_{\mathrm{sw}}^{\uparrow} \\
& =0 \\
\text { and } & \\
{ }^{\mathrm{sfc}} F_{\text {net }} & =-{ }^{\mathrm{sfc}} F_{\mathrm{lw}}^{\uparrow}-{ }^{\mathrm{sfc}} F_{\mathrm{sw}}^{\uparrow}+{ }^{\mathrm{sfc}} F_{\mathrm{lw}}^{\downarrow}+{ }^{\mathrm{sfc}} F_{\mathrm{sw}}^{\downarrow} \\
& =0,
\end{aligned}
$$

which gives

$$
\begin{aligned}
& T_{\mathrm{S}}=\sqrt[4]{\frac{S\left(1-\left(1-a_{\mathrm{S}}\right)\left(A\left(2-a_{\mathrm{S}}\right)-1\right)\right)}{\left(2-a_{1}\right) \sigma}} \approx 286 \mathrm{~K} \text { and } \\
& T_{\mathrm{a}}=\sqrt[4]{\frac{S-\left(1-a_{1}\right) \sigma T_{\mathrm{s}}^{4}-A\left(1-a_{\mathrm{S}}\right)^{2} S}{a_{1} \sigma}} \approx 255 \mathrm{~K} .
\end{aligned}
$$

The classical way to estimate the temperature caused by the greenhouse effect is to assume no atmosphere (i.e. $a_{1}=0$ 
and $a_{\mathrm{s}}=0$ ), which results in a surface temperature (Eq. 12) of around $255 \mathrm{~K}$. The difference of $31 \mathrm{~K}$ is then the effect of the atmosphere, i.e. the greenhouse effect.

For non-steady-state situations any flux imbalance leads to temperature changes, following

$$
\begin{aligned}
\frac{\partial T_{\mathrm{a}}}{\partial t} & =\frac{1}{c_{p}^{\text {air }} \rho^{\text {air }}} \frac{{ }^{\text {toa }} F_{\text {net }}-{ }^{\text {sfc }} F_{\text {net }}}{Z^{\text {air }}} \text { and } \\
\frac{\partial T_{\mathrm{s}}}{\partial t} & =\frac{1}{c_{p}^{\text {sfc }} \rho^{\text {sfc }}} \frac{{ }^{\text {sfc }} F_{\text {net }}}{Z^{\text {sfc }}} .
\end{aligned}
$$

The respective parameters are set to represent realistic values and to give a roughly $30 \mathrm{yr}$ response time of this simple climate system (Hasselmann et al., 1993). Changes in the concentration of carbon dioxide lead to a change in the absorption of longwave radiation. Therefore the absorption $a_{1}$ is parameterised in a very simplified way to allow principle studies on the greenhouse effect and on a doubling of $\mathrm{CO}_{2}$. The greenhouse gases are grouped (see also Sect. 2.1) into non-carbon dioxide ("nC"; e.g. water vapour, methane, nitrous oxide), base case carbon dioxide ("bC", here with a mixing ratio $C_{\mathrm{CO}_{2}}^{\mathrm{bC}}=360 \mathrm{ppmv}$ ) and enhanced carbon dioxide ("eC", e.g. $C_{\mathrm{CO}_{2}}^{\mathrm{eC}}=360$ ppmv for a doubling experiment). The absorptions are then defined as follows:

$$
\begin{aligned}
a_{1} & =a_{1}^{\mathrm{sw}}+a_{1}^{\mathrm{nC}}+a_{1}^{\mathrm{bC}}+a_{1}^{\mathrm{eC}}, \quad \text { with } \\
a_{1}^{\mathrm{sw}} & =0.0 \quad \text { (without atmosphere) } \\
a_{1}^{\mathrm{nC}} & =0.61 \\
a_{1}^{C} & =0.2-0.04 \frac{360 \mathrm{ppm}}{C_{\mathrm{CO}_{2}}} \\
a_{1}^{\mathrm{bC}} & =a_{1}^{C} \frac{C_{\mathrm{CO}_{2}}^{\mathrm{bC}}}{C_{\mathrm{CO}_{2}}} \\
a_{1}^{\mathrm{eC}} & =a_{1}^{C} \frac{C_{\mathrm{CO}_{2}}^{\mathrm{eC}}}{C_{\mathrm{CO}_{2}}} .
\end{aligned}
$$

The absorption with respect to $\mathrm{CO}_{2}$ depends on the total concentration: $C_{\mathrm{CO}_{2}}=C_{\mathrm{CO}_{2}}^{\mathrm{bC}}+C_{\mathrm{CO}_{2}}^{\mathrm{eC}}$ (see also Eq. 19). The individual contributions are then relative to the $\mathrm{CO}_{2}$ contribution.

An instantaneous doubling of the carbon dioxide concentration (from 360 to $720 \mathrm{ppmv}$ ) results in an increase in the longwave absorption from 0.77 to 0.79 with this absorption parameterisation. The resulting instantaneous radiative forcing ( ${ }^{\text {toa }} F_{\text {net }}$ ) according to Eq. (8) is $3.68 \mathrm{~W} \mathrm{~m}^{-2}$ leading to a steady-state temperature increase of $1.2 \mathrm{~K}$ and hence a climate sensitivity of $0.3 \mathrm{~K}\left(\mathrm{~W} \mathrm{~m}^{-2}\right)^{-1}$. Applying the method of Gregory et al. (2004) results in the identical value.

\subsection{Temperature tagging}

The temperature tagging aims at tracking temperature changes caused by any change in the climate system in every timestep of a simulation. In Sect. 2.1 the individual temperature categories are motivated. Here they are physically defined.

In the simple climate box model (Sect. 2.2), changes to the climate system are introduced via changes in the absorption, which are parameterised depending on the carbon dioxide concentration (Eq. 16). That means that the individual contributions $T_{\mathrm{a}}^{\mathrm{sw}}, T_{\mathrm{a}}^{\mathrm{nC}}, T_{\mathrm{a}}^{\mathrm{bC}}, T_{\mathrm{a}}^{\mathrm{eC}}$ to the total temperature $T_{\mathrm{a}}$ (and $T_{\mathrm{s}}$ in analogy) are dependent on and directly linked to the respective absorptions (Table 1).

The temperature equations are following Eqs. (14) and (15):

$$
\begin{aligned}
\frac{\partial T_{\mathrm{a}}^{i}}{\partial t} & =\frac{1}{c_{p}^{\text {air }} \rho^{\text {air }}} \frac{{ }^{\text {toa }} F_{\text {net }}^{i}-{ }^{\text {sfc }} F_{\text {net }}^{i}}{Z^{\text {air }}} \text { and } \\
\frac{\partial T_{\mathrm{s}}^{i}}{\partial t} & =\frac{1}{c_{p}^{\mathrm{sfc}} \rho^{\mathrm{sfc}}} \frac{{ }^{\mathrm{sfc}} F_{\text {net }}^{i}}{Z^{\mathrm{sfc}}}
\end{aligned}
$$

with $i \in\{\mathrm{sw}, \mathrm{nC}, \mathrm{bC}, \mathrm{eC}\}$. Hence the heart of the temperature tagging is to derive the appropriate fluxes associated with the absorption of the individual components.

In this set-up, all shortwave fluxes are attributed to one component only ("sw", Table 1). Therefore, the tagged shortwave fluxes are straightforward:

$$
\begin{aligned}
& { }^{\text {toa }} F_{\mathrm{sw}}^{\uparrow}{ }^{\mathrm{sw}}={ }^{\text {toa }} F_{\mathrm{sw}}^{\uparrow} \\
& { }^{\text {toa }} F_{\mathrm{sw}}^{\downarrow}{ }^{\mathrm{sw}}={ }^{\text {toa }} F_{\mathrm{sw}}^{\downarrow} \\
& { }^{\mathrm{sfc}} F_{\mathrm{sw}}^{\uparrow}{ }^{\mathrm{sw}}={ }^{\mathrm{sfc}} F_{\mathrm{sw}}^{\uparrow} \\
& { }^{\mathrm{sfc}} F_{\mathrm{sw}}^{\downarrow}{ }^{\mathrm{sw}}={ }^{\mathrm{sfc}} F_{\mathrm{sw}}^{\downarrow},
\end{aligned}
$$

whereas for all other categories the shortwave fluxes are zero.

The only non-trivial parts of separation of fluxes into their contributions are the longwave components. To illustrate the main idea, which is following the tagging principle for chemical reactions (Grewe et al., 2010), the term $\left(1-a_{1}\right)^{\mathrm{sfc}} F_{1 \mathrm{w}}^{\uparrow}$ as part of the flux ${ }^{\text {toa }} F_{1 \mathrm{w}}^{\uparrow}$ is considered in more detail in the following. This is a typical example of a non-linear interaction of multiple categories, where both the longwave absorption and the flux ${ }^{\mathrm{sfc}} F_{\mathrm{lw}}^{\uparrow}$ have contributions from individual categories. This term can be written as

$$
\begin{aligned}
& \left(1-a_{1}\right)^{\mathrm{sfc}} F_{\mathrm{lw}}^{\uparrow} \\
& =\left(1-\sum_{i} a_{1}^{i}\right) \sum_{i}{ }^{\mathrm{sfc}} F_{\mathrm{lw}}^{\uparrow} \\
& =\sum_{i}{ }^{\mathrm{sfc}} F_{\mathrm{lw}}^{\uparrow}-\sum_{i, j} a_{1}^{i}{ }^{\mathrm{sfc}} F_{\mathrm{lw}}^{\uparrow} j
\end{aligned}
$$




$$
\begin{aligned}
& =\sum_{i}\left(1-a_{1}^{i}\right){ }^{\mathrm{sfc}} F_{1 \mathrm{w}}^{\uparrow}-\sum_{i \neq j} a_{1}^{i}{ }^{\mathrm{sfc}} F_{1 \mathrm{w}}^{\uparrow j} \\
& =\sum_{i}\left(1-a_{1}^{i}\right){ }^{\mathrm{sfc}} F_{\mathrm{lw}}^{\uparrow}-\frac{1}{2} \sum_{i \neq j} a_{1}^{i}{ }^{\mathrm{sfc}} F_{\mathrm{lw}}^{\uparrow}{ }^{j} \\
& -\frac{1}{2} \sum_{i \neq j} a_{1}^{i \text { sfc }} F_{1 \mathrm{w}}^{\uparrow j} \\
& =\sum_{i}\left[\left(1-a_{1}^{i}\right){ }^{\mathrm{sfc}} F_{\mathrm{lw}}^{\uparrow}{ }^{i}-\frac{1}{2} a_{1}^{i}\left({ }^{\mathrm{sfc}} F_{\mathrm{lw}}^{\uparrow}-{ }^{\mathrm{sfc}} F_{\mathrm{lw}}^{\uparrow}{ }^{i}\right)\right. \\
& \left.-\frac{1}{2}\left(a_{1}-a_{1}^{i}\right){ }^{\mathrm{sfc}} F_{1 \mathrm{w}}^{\uparrow}{ }^{i}\right] \\
& =\sum_{i}\left[{ }^{\mathrm{sfc}} F_{\mathrm{lw}}^{\uparrow}{ }^{i}-\frac{1}{2}\left(a_{1}^{i \mathrm{sfc}} F_{\mathrm{lw}}^{\uparrow}+a_{1}{ }^{\mathrm{sfc}} F_{\mathrm{lw}}^{\uparrow}\right)\right] .
\end{aligned}
$$

The non-linearity in this term arises from $\sum a_{i} F_{j}$, the second term in Eq. (30), which describes the flux of category $j$ absorbed by category $i$. For example, what happens when the radiation emitted at the surface associated with the "sw" radiation is absorbed in the atmosphere by the enhanced $\mathrm{CO}_{2}$ concentration ("eC")? The argument is that both processes are equally important and hence contribute equally $50 \%$ to the fluxes ${ }^{\text {sfc }} F_{\text {lw }}^{\uparrow}$ and ${ }^{\text {sfc }} F_{1 \mathrm{w}}^{\uparrow j}$ (Eq. 31). Note that this is the basic concept of this methodology. Whenever two processes or quantities are necessarily required, then they are equally important and contribute $50 \%$ to non-linearities. Hence, we find a decomposition of the left-hand side of Eq. (28) and can define the contribution of category $i$ to the flux $\left(1-a_{1}\right){ }^{\mathrm{sfc}} F_{\text {lw }}^{\uparrow}$ by

$$
\begin{aligned}
& \left(1-a_{1}\right)^{\mathrm{sfc}} F_{\mathrm{lw}}^{\uparrow} \\
& =\sum_{i}[\underbrace{\mathrm{sfc}^{\mathrm{F}_{\mathrm{lw}}}{ }^{i}}_{\text {flux } i} \underbrace{-\frac{1}{2} a_{1}^{i} \mathrm{sfc}^{\mathrm{sfc}} F_{\mathrm{lw}}^{\uparrow}}_{\begin{array}{c}
\text { total flux } \\
\text { absorbed by } i
\end{array}} \underbrace{-\frac{1}{2} a_{1}{ }^{\mathrm{sfc}} F_{\mathrm{lw}}^{\uparrow}{ }^{i}}_{\begin{array}{c}
\text { flux } i \text { absorbed } \\
\text { by all }
\end{array}}]
\end{aligned}
$$

or alternatively

$$
=\sum_{i}\left[{ }^{\mathrm{sfc}} F_{\mathrm{lw}}^{\uparrow}{ }^{i}-\frac{1}{2} a_{1}{ }^{\mathrm{sfc}} F_{\mathrm{lw}}^{\uparrow}\left(\frac{a_{1}^{i}}{a_{1}}+\frac{{ }^{\mathrm{sfc}} F_{\mathrm{lw}}^{\uparrow}}{\mathrm{sfc}^{i} F_{\mathrm{lw}}^{\uparrow}}\right)\right] .
$$

The contribution from category $i$ to the longwave flux at the top of the atmosphere resulting from the emission at the surface consists of 3 parts (Eq. 34): (1) the contribution of category $i$ to the emitted flux, i.e. ${ }^{{ }^{\mathrm{fcc}}} F_{\mathrm{lw}}^{\uparrow}{ }^{i}$; (2) the reduction of this flux by the contribution of category $i$ to the absorption; and (3) the reduction of this flux by the total absorption of flux $i$. The factor $1 / 2$ means that the other $1 / 2$ of this contribution is assigned to another category (see also above). The terms (2) and (3) both include the contribution $a_{1}^{i} \operatorname{sfc}^{F_{1 \mathrm{w}}{ }^{i}}$ of $50 \%$ and hence provide together the total.

The alternative formulation (Eq. 35) shows that the tagged absorbed flux can also be written as a fraction of the total absorbed flux $a_{1}{ }^{\mathrm{sfc}} F_{\mathrm{lw}}^{\uparrow}$. The fraction is the mean of the relative contribution of the tagged absorption and the tagged longwave upward flux at the surface.

The top of the atmosphere longwave flux ${ }^{\text {toa }} F_{\text {lw }}^{\uparrow}$ consists of two parts (see Eq. 7). The transmittance has just been discussed above, and the flux emitted by the atmosphere is

$\frac{T_{\mathrm{a}}^{i}}{T_{\mathrm{a}}} a_{1} \sigma T_{a}^{4}=a_{1} \sigma T_{\mathrm{a}}^{3} T_{\mathrm{a}}^{i}$.

Here the basic idea is that the total temperature is relevant for the radiation emission and that no contribution is more important than the other, which directly leads to a linear decomposition according to the individual contributions $T_{\mathrm{a}}^{i}$ to the atmospheric temperature $T_{\mathrm{a}}$. Note that this is a different approach than considered in a perturbation approach, where a temperature change would be diagnosed for the case that the absorption is changed. This would give a temperature sensitivity, whereas the tagging provides consistent temperature contributions. This is often confused but has to be distinguished, since both approaches differ significantly and address different questions (Grewe et al., 2010, 2012; Grewe, 2013).

Putting all this together, the tagged fluxes are

$$
\begin{aligned}
& { }^{\mathrm{sfc}} F_{\mathrm{lw}}^{\uparrow}{ }^{i}=\frac{T_{\mathrm{s}}^{i}}{T_{\mathrm{s}}}{ }^{\mathrm{sfc}} F_{\mathrm{lw}}^{\uparrow} \\
& { }^{\mathrm{sfc}} F_{\mathrm{lw}}^{\downarrow}{ }^{i}={ }^{\operatorname{sfc}} F_{1 \mathrm{w}} \frac{1}{2}\left(\frac{T_{\mathrm{a}}^{i}}{T_{\mathrm{a}}}+\frac{a_{1}^{i}}{a_{\mathrm{l}}}\right) \\
& { }^{\text {toa }} F_{\mathrm{lw}}^{\uparrow^{i}}={ }^{\mathrm{sfc}} F_{\mathrm{lw}}^{{ }^{i}}-\frac{1}{2} a_{1}^{\mathrm{sfc}} F_{\mathrm{lw}}^{\uparrow}\left(\frac{a_{1}^{i}}{a_{1}}+\frac{\mathrm{sfc}^{{ }^{\uparrow}} F_{\mathrm{lw}}^{i}}{\mathrm{sfc}_{\mathrm{lf}_{\mathrm{lw}}}^{\uparrow}}\right) \\
& +{ }^{\mathrm{sfc}} F_{\mathrm{lw}} \frac{1}{2}\left(\frac{T_{\mathrm{a}}^{i}}{T_{\mathrm{a}}}+\frac{a_{1}^{i}}{a_{\mathrm{l}}}\right) .
\end{aligned}
$$

By definition, the sum of all tagged fluxes equals the total flux, and hence the sum of the tagged temperatures equals the total temperature:

$\sum_{i} T_{\mathrm{a}}^{i}=T_{\mathrm{a}} \quad$ and
$\sum_{i} T_{\mathrm{s}}^{i}=T_{\mathrm{s}}$.

This means that the definition of the categories leads to a complete decomposition of the temperature as required.

\subsection{Experimental set-up}

The climate box model is formulated in Fortran90, with a timestep of 1 day. The temperatures are initialised by the steady-state values and the tagged temperatures by equal shares of the respective temperatures. A spin-up of $1000 \mathrm{yr}$ is regarded, before the actual experiment starts with a simulation length of $550 \mathrm{yr}$ to again obtain an equilibrium situation.

For the greenhouse effect experiments, the absorption of the atmosphere is varied in steps of $\alpha=0.0,0.1, \ldots, 0.9,1.0$; 
i.e. a shortwave and a longwave absorption of $\alpha a_{\mathrm{s}}$ and $\alpha a_{1}$ are taken into account ( $a_{1}^{\mathrm{bC}}=0.16$ and $\left.a_{1}^{\mathrm{eC}}=0\right) . \alpha=0$ and $\alpha=1$ refer to "no atmosphere" and "full atmosphere", respectively.

The $\mathrm{CO}_{2}$ doubling experiment starts with a background $\mathrm{CO}_{2}$ concentration of $360 \mathrm{ppmv}\left(C_{\mathrm{CO}_{2}}^{\mathrm{bC}}=360 \mathrm{ppmv}\right.$ and $C_{\mathrm{CO}_{2}}^{\mathrm{eC}}=0$ ppmv) for the spin-up period. After the spin-up $C_{\mathrm{CO}_{2}}^{\mathrm{eC}}$ is set to $360 \mathrm{ppmv}\left(a_{1}^{\mathrm{eC}}=a_{1}^{\mathrm{bC}}=0.09\right)$.

\section{Results}

\subsection{The greenhouse effect}

In Sect. 2.2 the greenhouse effect is defined as the difference in surface temperature for the cases with and without an atmosphere (Andrews, 2010; Barry and Chorley, 2003; Jacobsen, 2002; Ramanathan, 1998; Smil, 2003). Another explanation is given by IPCC (2007, p. 946): "Greenhouse gases effectively absorb thermal infrared radiation, emitted by the Earth's surface, by the atmosphere itself due to the same gases, and by clouds. Atmospheric radiation is emitted to all sides, including downward to the Earth's surface. Thus, greenhouse gases trap heat within the surface-troposphere system. This is called the greenhouse effect."

These are two definitions, which basically address the same physical properties of the atmosphere. However, they are inconsistent as will be shown in the following.

Figure 2a shows the increase in surface temperature when continuously increasing the atmospheric absorption (both sw and $\mathrm{lw}$ ) from no atmosphere to the full atmosphere, reaching roughly $30 \mathrm{~K}$, consistent with, for example, Andrews (2010). Figure $2 \mathrm{~b}$ shows the contributions to the surface temperature from the shortwave component (red), non- $\mathrm{CO}_{2}$ greenhouse gases (green) and from $\mathrm{CO}_{2}$ (blue). The contribution of greenhouse gases (red and blue) is $68 \mathrm{~K}$ and hence a factor of two larger than the classical calculation of the greenhouse effect (Fig. 2a).

One major difference is the different handling of the shortwave absorption of the atmosphere. In Fig. 2a the transition from no to a full atmosphere includes an increase in both the shortwave absorption and the longwave absorption, whereas in Fig. $2 \mathrm{~b}$ the temperature associated with greenhouse gases $\left(T^{\mathrm{nC}}+T^{\mathrm{bC}}+T^{\mathrm{eC}}\right)$ is solely attributed to longwave absorption. Switching from no to a full atmosphere leads to an increase in the shortwave absorption and consequently to a decrease of the shortwave downward and upward radiation at the surface (Fig. 3a). The net surface shortwave radiation ( ${ }^{\mathrm{sfc}} F_{\mathrm{sw}}^{\downarrow}-{ }^{\mathrm{sfc}} F_{\mathrm{sw}}^{\uparrow}$ ) is decreasing by around $50 \mathrm{~W} \mathrm{~m}^{-2}$. This decrease in the flux leads to a decrease of the surface temperature by roughly $14 \mathrm{~K}$ (Fig. $2 \mathrm{~b}$, solid line). It can be easily determined by considering a surface flux balance for an atmosphere without a longwave absorption $\left(a_{1}=0\right)$, but with a shortwave absorption, which gives a temperature of $\sqrt[4]{\left(1-A\left(1-a_{\mathrm{s}}\right)^{2}\right) S / \sigma}$.

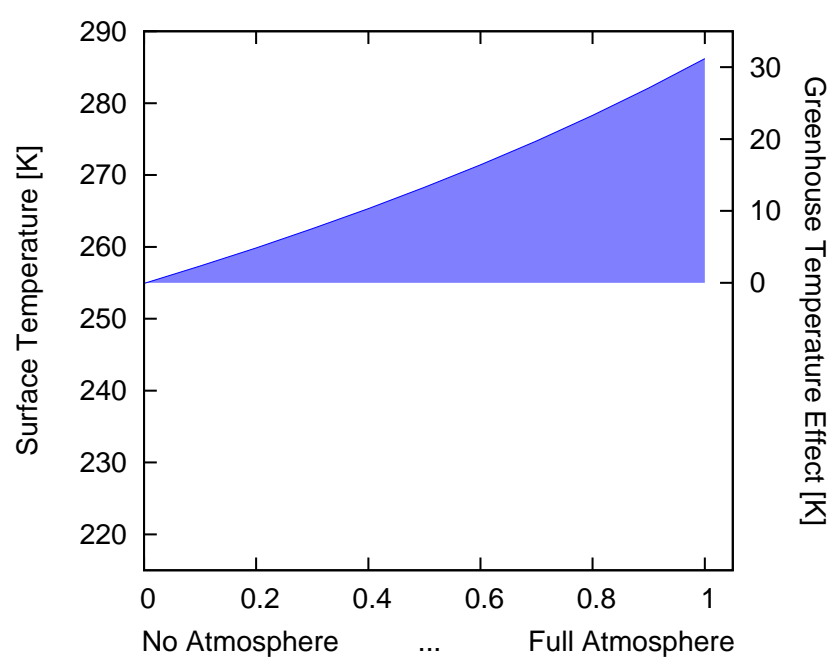

(a)

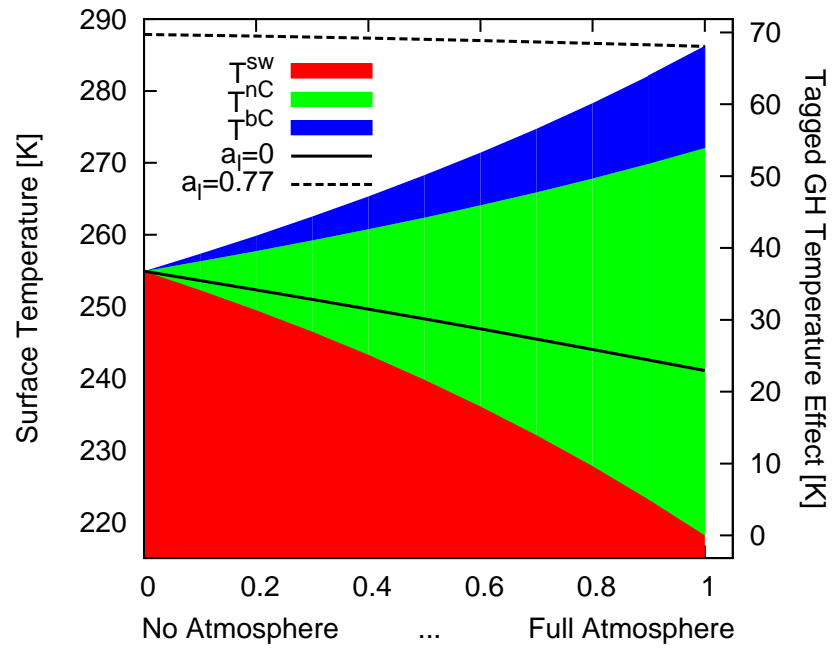

(b)

Fig. 2. Top: steady-state surface temperature $(\mathrm{K})$ for the situation of no atmosphere $(=0)$ to a full atmosphere $(=1)$. In between the shortwave and longwave absorption are scaled. Bottom: as top but with additional information of the contribution of the longwave absorption due to non- $\mathrm{CO}_{2}$ greenhouse gases ("nC", red) and $\mathrm{CO}_{2}$ ("bC", green). The temperature $T_{\mathrm{S}}^{\mathrm{sw}}$ follows the lower edge of the red areas. Additionally two sensitivity experiments are included, where longwave absorption is excluded, which gives the effect of shortwave absorption on the surface temperature (solid line) and where longwave absorption is set to the "full atmosphere value" of $a_{1}=0.77$ (dashed line).

This shortwave cooling effect is largely compensated by an increased longwave absorption of the atmosphere. This can be shown in a simple experiment, where the longwave absorption is constant $\left(a_{1}=0.77\right)$ and only the shortwave absorption varies (Fig. 2b, dashed line). The compensating effect leads to an increase in atmospheric temperatures and an increase in the longwave downward flux at the surface of more than $200 \mathrm{Wm}^{-2}$ (Fig. 3a, blue dotted line). The 

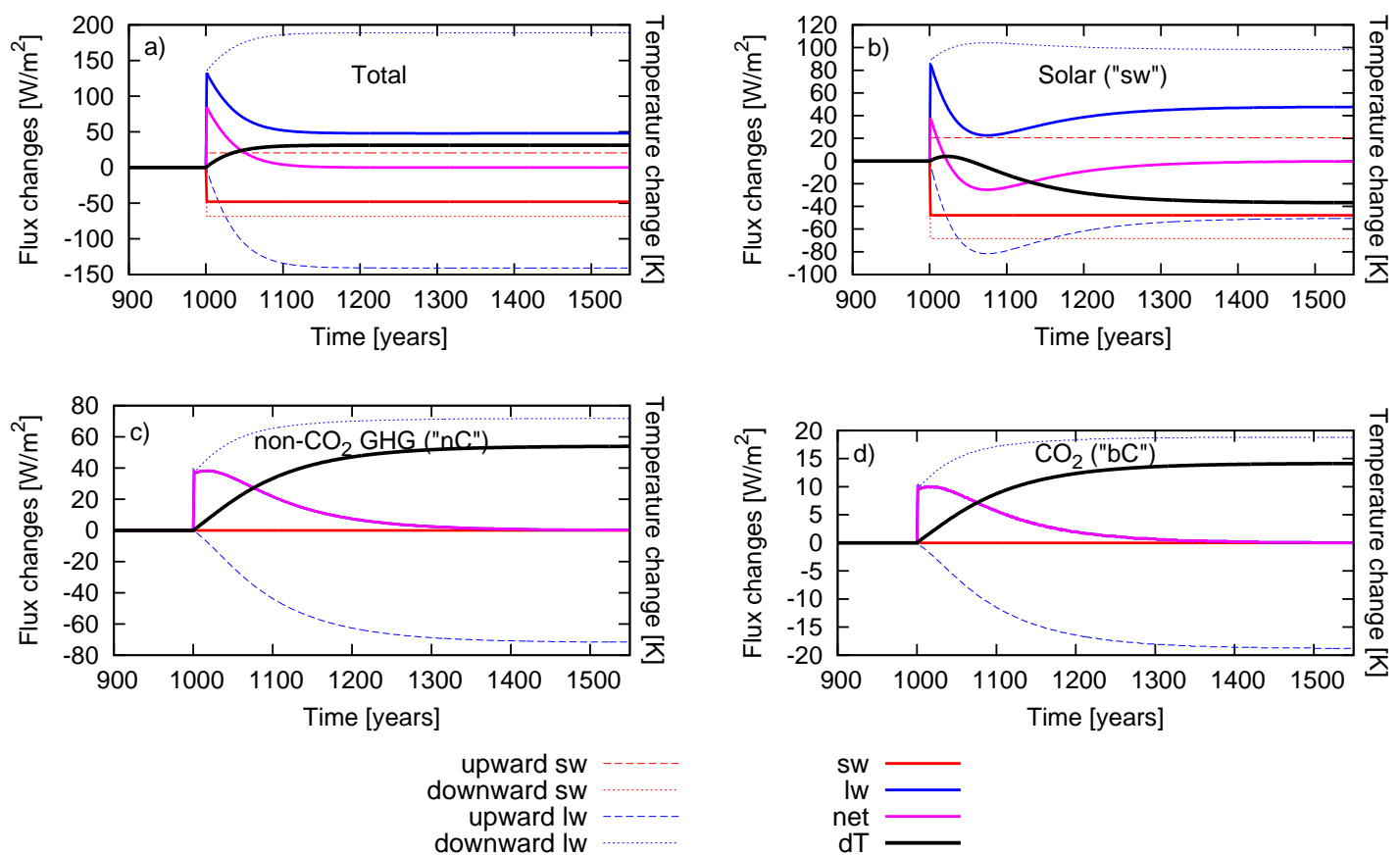

Fig. 3. Evolution of flux changes $\left(\mathrm{Wm}^{-2}\right)$ (coloured lines) and temperature changes $(\mathrm{K})$ (black line) in the experiment, where the shortwave and longwave absorption is zero during the spin-up time (1000 yr) and then set to the "full atmosphere" values $\left(a_{\mathrm{S}}=0.2\right.$ and $\left.a_{1}=0.77\right)$. Shortwave fluxes are shown in red, longwave in blue. Upward (negative) and downward (positive) fluxes are dashed and dotted, respectively. The total net-flux changes are shown as a purple solid line. Changes are shown for the total flux and temperature changes (a) and the individual tagging categories: "sw" (b), "nC" (c), and "bC"(d).

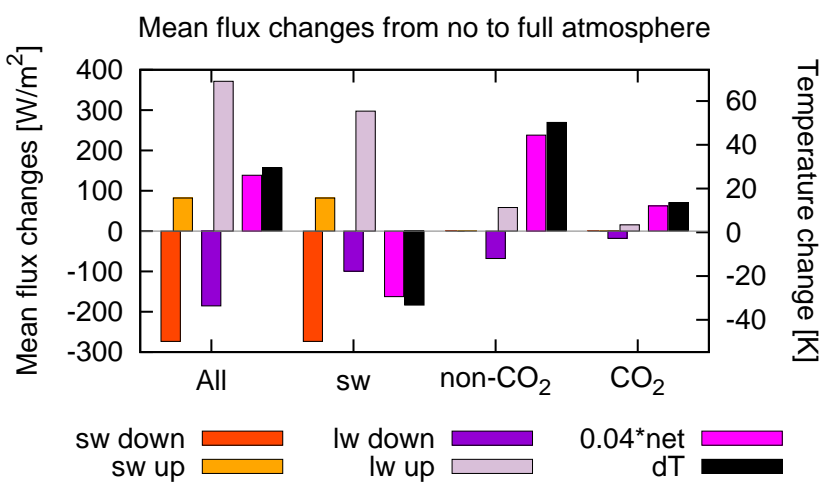

Fig. 4. Summary of Fig. 3. Mean flux changes over the simulation period are shown for the total ("All") and the regarded tagging categories. Shortwave and longwave fluxes are shown in red and blue, respectively. Downward fluxes are shown in darker colour than upward fluxes. The net flux (purple) is scaled for presentation reasons. Temperature changes are in black.

net longwave warming effect at the surface is largely overcompensating the shortwave cooling effect, leading to a positive total flux and an increase of the surface temperature (Fig. 4).
Therefore, during the transition from no atmosphere to a full atmosphere, the temperature associated with the shortwave radiation $\left(T_{\mathrm{S}}^{\mathrm{sw}}\right)$ decreases, since the shortwave cooling is, different to the surface temperature $\left(T_{\mathrm{S}}\right)$, not overcompensated by longwave radiation (Figs. $3 b$ and 4 ). The upward longwave radiation at the surface is attributed to the category "sw" by $100 \%$ in the case of no atmosphere. Hence also the upward longwave flux at the top of the atmosphere and the downward flux at the surface are fully attributed to the category "sw". The greenhouse gases (categories "nC" and "bC") absorb longwave radiation and contribute considerably to the longwave atmospheric radiation leading to an increase in temperature of $68 \mathrm{~K}$. Since the processes are identical for the categories " $\mathrm{nC}$ " and "bC", the results differ only by a constant factor (Figs. 3c, d and 4).

Figure 5 summarises the results by comparing the net shortwave flux and the downward longwave flux. Their sum has to be balanced by the emitted longwave radiation at the surface temperature (Eq. 11). Without an atmosphere (left) the surface temperature $(255 \mathrm{~K})$ is determined by the net shortwave radiation (see also above). With an atmosphere, which absorbs only in the shortwave, this shortwave flux is reduced and leads to a surface temperature of $241 \mathrm{~K}$. With an atmosphere (second right) the longwave downward flux adds to the net shortwave flux by approximately the same amount 


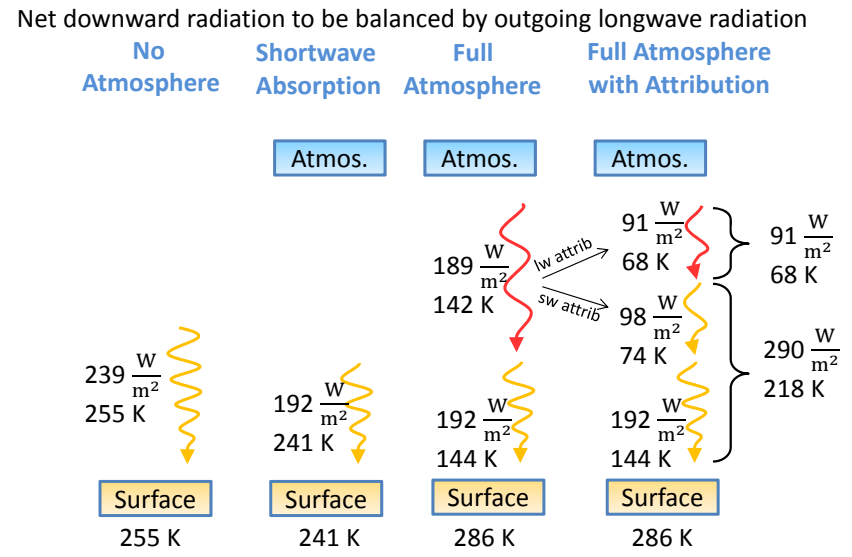

Fig. 5. Shortwave (net) and longwave (downward) fluxes at the surface $\left(\mathrm{W} \mathrm{m}^{-2}\right)$ and respective surface temperature contribution $(\mathrm{K})$ for the case without an atmosphere (left) with an atmosphere only absorbing in the shortwave (second left), with a full atmosphere (second right) and additionally temperatures attributed to longwave and shortwave radiation. The surface temperature leads to an upward longwave radiation, which has to be balanced by the net shortwave and longwave fluxes.

and increases the surface temperature to $286 \mathrm{~K}$. Hence the contribution of the net shortwave flux and longwave flux to the surface temperature are $144 \mathrm{~K}$ and $142 \mathrm{~K}$, respectively. However this is not yet the temperature associated with the shortwave and longwave processes, since a large fraction of the longwave radiation, which is emitted by the surface, is actually caused by shortwave radiation. This radiation is absorbed by the atmosphere and contributes to the longwave downward radiation. Hence the total longwave downward radiation of $189 \mathrm{~W} \mathrm{~m}^{-2}$ has a contribution of $98 \mathrm{~W} \mathrm{~m}^{-2}$ due to the radiation emitted by the surface (the part which was heated by solar radiation) and a second contribution of $91 \mathrm{~W} \mathrm{~m}^{-2}$ due to absorption of longwave radiation by greenhouse gases (right part of Fig. 5). This attribution is shown in more detail in Fig. 6. The temperature increases highly non-linearly (4th square root) with the surface flux. In the situation of an atmosphere absorbing only in the shortwave, the surface flux of $192 \mathrm{Wm}^{-2}$ leads to a temperature of $241 \mathrm{~K}$ (red). The temperature to flux ratio (solid line) is much lower in the situation of a full atmosphere, and the flux of $381 \mathrm{~W} \mathrm{~m}^{-2}$ leads to a temperature of $286 \mathrm{~K}$. Due to this lower ratio, the flux of $192 \mathrm{~W} \mathrm{~m}^{-2}$ leads to a much lower contribution to the surface temperature of $144 \mathrm{~K}$ (red) in the situation of a full atmosphere. The flux originating from the solar heating of the surface and the subsequent longwave radiation and atmospheric absorption amounts to $98 \mathrm{~W} \mathrm{~m}^{-2}$ and thus leading to $74 \mathrm{~K}$ (green). The remaining greenhouse effect, the absorption of longwave radiation due to greenhouse gases, accounts for a flux of $91 \mathrm{~W} \mathrm{~m}^{-2}$ and hence $68 \mathrm{~K}$ (blue).

Therefore, the two different ways to calculate the contribution of the greenhouse effect to the surface temperature of

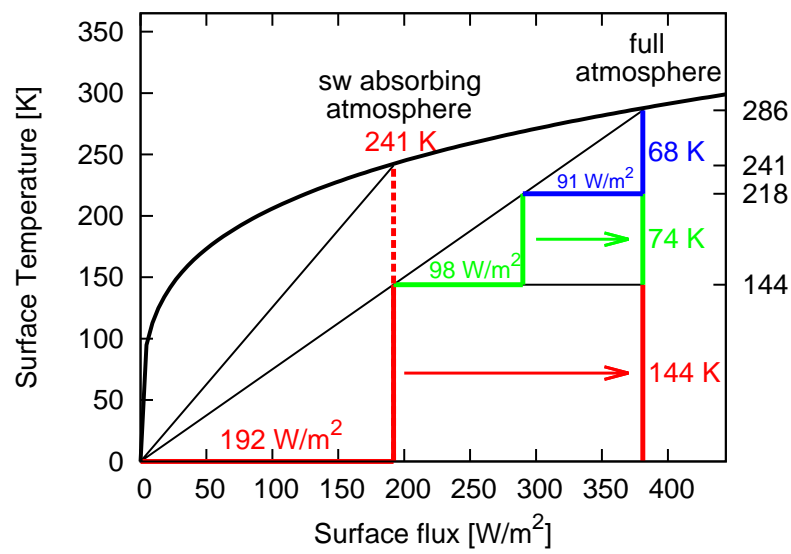

Fig. 6. Surface temperature $(\mathrm{K})$ as a function of the surface flux (sum of net sw and downward lw) (black line $\mathrm{W} \mathrm{m}^{-2}$ ) and attribution of temperature contributions to fluxes (colour code as in Fig. 2: red: sw contribution; green: background greenhouse gases; and blue background: $\mathrm{CO}_{2}$ ). See also Fig. 5 .

$31 \mathrm{~K}$ and $68 \mathrm{~K}$ largely differ. The surface temperature without an atmosphere is $255 \mathrm{~K}$ (Fig. 5). The surface fluxes are reduced for an atmosphere, which solely absorbs in the shortwave. The surface temperature decreases by $14 \mathrm{~K}$ to $241 \mathrm{~K}$. The surface flux increases by a factor of two because of the longwave absorption, if a full atmosphere is considered. This leads to a reduction of the contribution of the shortwave influx to the surface temperature from $100 \%$ to $75 \%$, when changing from an atmosphere which only absorbs in the shortwave to a full atmosphere. This contribution of roughly $75 \%$, which represents a flux of $290 \mathrm{~W} \mathrm{~m}^{-2}$, leads to a temperature $T_{\mathrm{s}}^{\mathrm{sw}}$ of $218 \mathrm{~K}$ and a temperature associated with the greenhouse effect of $T_{\mathrm{s}}^{\mathrm{nC}}+T_{\mathrm{s}}^{\mathrm{bC}}=68 \mathrm{~K}$.

\subsection{Effects of a $\mathrm{CO}_{2}$ doubling}

Figure 7 shows the top of the atmosphere flux, surface and temperature changes for the $\mathrm{CO}_{2}$ doubling experiment. After the spin-up time, the radiation flux responds immediately to the change in the longwave absorption. The atmospheric temperature shows an immediate drop. This drop is caused by the different response times of the atmosphere and the surface. Shortly after the change in the absorption, the surface fluxes are almost unaffected, but the atmospheric temperatures react quickly. Hence the atmospheric longwave emission increases, leading to a radiative cooling. Then the surface temperature slowly increases due to the increasing flux emitted from the atmosphere caused by the increased absorption, which in turn also leads to a warming of the atmosphere.

The contribution of the greenhouse gases to the surface temperature is shown in Fig. 8. The largest contribution with $218 \mathrm{~K}$ arises from the incoming shortwave radiation heating the surface $\left(T_{\mathrm{s}}^{\mathrm{sw}}\right)$ as already discussed in Sect. 3.1. The non$\mathrm{CO}_{2}$ greenhouse gases contribute with additional $55 \mathrm{~K}\left(T_{\mathrm{s}}^{\mathrm{nC}}\right)$ 


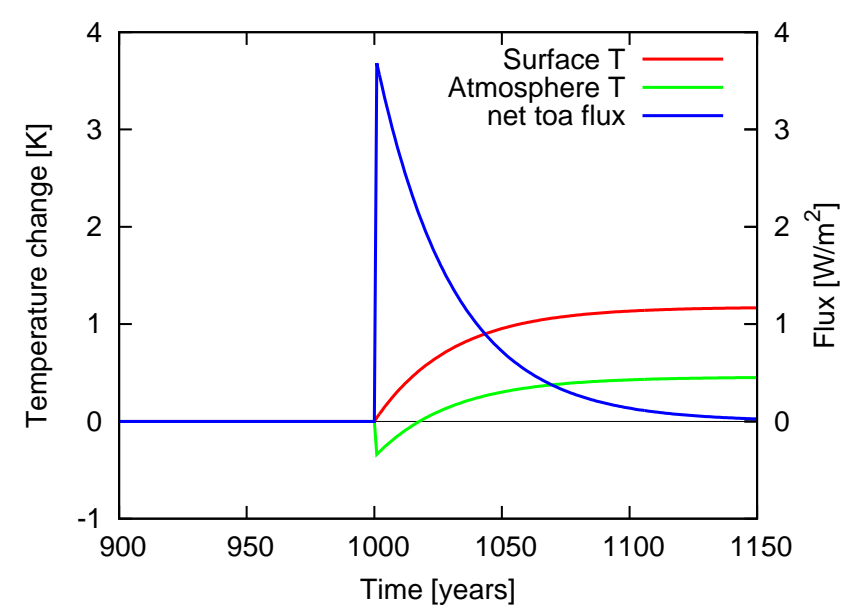

Fig. 7. Temporal development of the top of the atmosphere flux $\left({ }^{\text {toa }} F_{\text {net }}\right)$ in $\mathrm{W} \mathrm{m}^{-2}$, surface and atmosphere temperature $\left(T_{\mathrm{S}}\right.$ and $T_{\mathrm{a}}$ ) in an experiment, where after a $1000 \mathrm{yr}$ spin-up $\mathrm{CO}_{2}$ concentration is doubled.

and the background $\mathrm{CO}_{2}$ concentration with around $15 \mathrm{~K}$ $\left(T_{\mathrm{s}}^{\mathrm{bC}}\right)$. The change in $\mathrm{CO}_{2}$ concentration leads to changes in the surface temperature $T_{\mathrm{s}}$, which largely can be explained by the change in the temperature associated with the $\mathrm{CO}_{2}$ change $\left(T_{\mathrm{s}}^{\mathrm{bC}}+T_{\mathrm{s}}^{\mathrm{eC}}\right)$ (Fig. 9). The temperature of the individual $\mathrm{CO}_{2}$ contributions $T_{\mathrm{s}}^{\mathrm{bC}}$ and $T_{\mathrm{s}}^{\mathrm{eC}}$ are identical with around $8 \mathrm{~K}$ when reaching steady state for the $\mathrm{CO}_{2}$ doubling experiment. They have to be identical, since the base $\mathrm{CO}_{2}$ concentration and the enhanced concentration are equal and radiation does not favour any kind. Note also that the perturbation lifetime of the tagged temperatures is significantly larger (90 yr) compared to the perturbation lifetime of the climate system of around $30 \mathrm{yr}$.

The increase of atmospheric longwave absorption leads to an increase in the atmospheric temperature. This increase is not caused by the category "sw", and hence the ratio $T_{\mathrm{a}}^{\mathrm{sw}} / T_{\mathrm{a}}$ decreases, meaning that the contribution of the shortwave component to the atmospheric temperature decreases. Hence the longwave downward flux associated with the "sw" component decreases, leading to a lower surface temperature associated with the category "sw". (This mechanism is identical to the longwave contribution in the decrease of $T_{\mathrm{s}}^{\mathrm{sw}}$ from a "no atmosphere" to a "full atmosphere" scenario. See previous section.)

The increase in the surface temperature also leads to an enhanced upward longwave surface flux, which in turn increases the absorption of the non- $\mathrm{CO}_{2}$ greenhouse gases leading to an increase of the temperature $T_{\mathrm{s}}^{\mathrm{nC}}$.

Hence, this tagging methodology nicely allows a separation of non-linear effects. A doubling of the $\mathrm{CO}_{2}$ concentration in this climate box model leads to an increase in the $\mathrm{CO}_{2}$-related temperature of around $1.9 \mathrm{~K}$, enhancing the overall greenhouse effect and hence also the contribution of non- $\mathrm{CO}_{2}$ greenhouse gases by around $0.6 \mathrm{~K}$, whereas

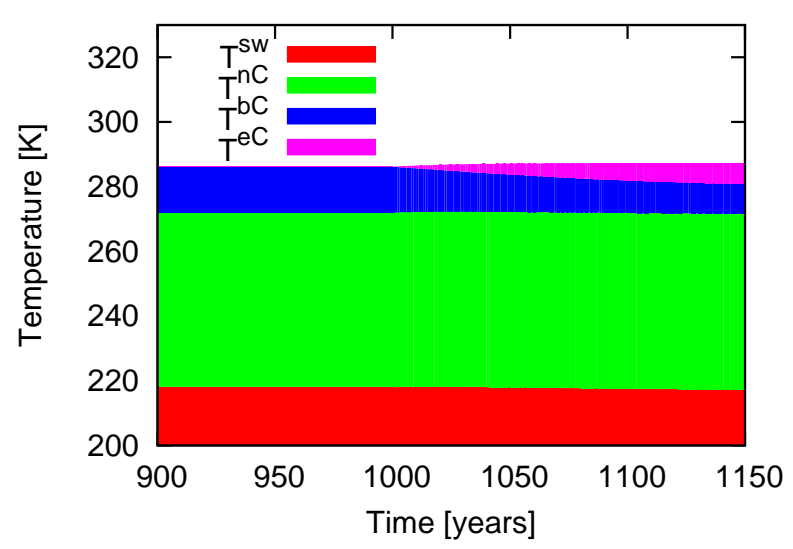

Fig. 8. Temporal development surface temperature and the individual tagged surface temperatures $(\mathrm{K})$ in an experiment, where after a $1000 \mathrm{yr}$ spin-up $\mathrm{CO}_{2}$ concentration is doubled.

the temperature associated with shortwave solar input is decreased by roughly $1.3 \mathrm{~K}$. (Note that these numbers refer to steady state, i.e. after $500 \mathrm{yr}$.) Therefore, a temperature increase of $2.5 \mathrm{~K}$ from the greenhouse effect is reduced by $1.3 \mathrm{~K}$ due to the decreased importance of the shortwave components ("sw"), leading to an overall increase in temperatures of around $1.2 \mathrm{~K}$.

\section{Towards a temperature tagging in a comprehensive climate model - some remarks on the method and applications}

The method of temperature tagging presented here is applied to a very simple box climate model. It shows the feasibility of tagging temperature (or any other quantity). In comprehensive climate models the temperature equation includes advection, adiabatic heating, diffusion, and diabatic heating, e.g. radiation and latent heat release. A detailed description of a temperature tagging in such a model is beyond the scope of this paper. However, I like to give some ideas on possible implementations. A generalized tagging approach is presented in a companion paper (Grewe, 2013).

Figure 10 gives an simplified overview on an arbitrary 3D climate model (black) and necessary extensions for the tagging scheme (red). First, quantities are defined and initialised. Without loss of generality, temperature is regarded here as a tracer, i.e. an advected quantity. For the tagging scheme additional tracers have to be defined and initialised accordingly. For the Russian heat wave example, this would imply two tracers: one which experiences all natural effects and one with anthropogenic greenhouse gas effects. The sum of both fields equals the temperature field. These fields are then advected by the models's advection scheme. During the physics and radiation calculation, all temperature tendencies have to be extracted and tagging tendencies calculated from these tendencies, which is basically the heart of the tagging 


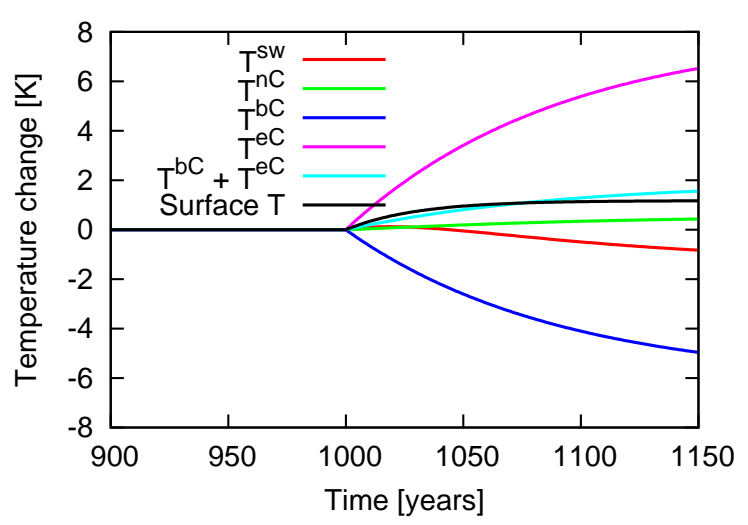

Fig. 9. As Fig. 8, but for changes relative to the year 1000, i.e. before the $\mathrm{CO}_{2}$ doubling.

scheme and refers to Sect. 2.3 in this paper and to Grewe (2013). Finally the temperature field and the tagged fields have to be integrated; i.e. the next time step value is calculated based on the tendencies. This leads to two 3-D fields for every timestep, which give the contribution (in K) of natural and anthropogenic effects to temperature.

The easiest part of the temperature equation is advection, since advection is a linear operator. Hence applying the advection scheme to the individual tagged temperature fields gives in the sum, if the advection scheme is mass conserving, the same result as the advection of the sum, i.e. the temperature. Also straightforward is the handling of the adiabatic heating term in the tagging equation. This is a process which affects all tagged temperature fields equally, and hence the adiabatic heating term of the temperature equation can be linearly decomposed according to the contribution of the individual tagged temperatures to the total temperature.

The more complex part of the temperature tagging equation includes the diabatic terms, as already in this application. It might be worth considering individual tagging categories for these terms, like temperature from latent heat, and (as in this application) from individual radiation components. For the latter, the heating rates caused by individual components have to be determined. A companion paper (Grewe, 2013) describes a generalized tagging formula and provides a basis for how to treat more complex processes.

An implementation of a temperature tagging would provide a method to answer questions like how much of the Russian heat wave is caused by man-made $\mathrm{CO}_{2}$ emissions (see also Introduction). This question must not be confused with another frequently asked question - namely whether this event would have happened in a world without climate change - which cannot be answered by the tagging method.

Other applications might be the quantification of feedback processes, like the temperature change caused by ozone depletion. How large is the contribution to tropospheric temperatures? The large advantage of this method is its diagnostic nature. There are hardly any aspects of statistical noise.

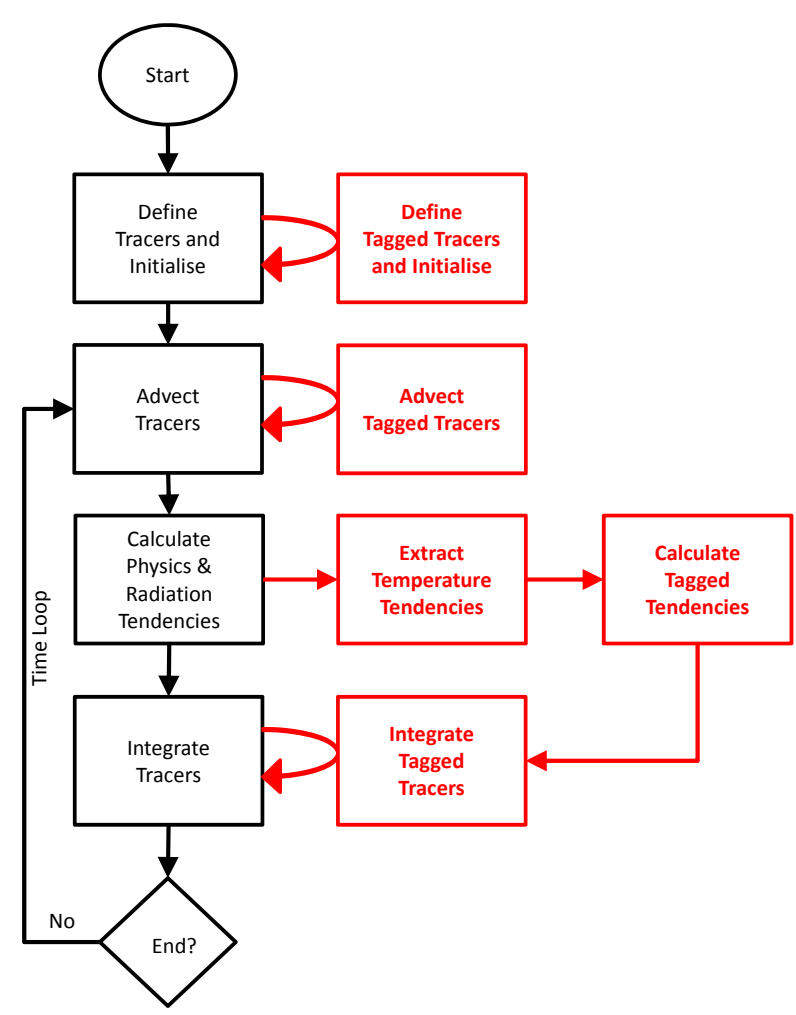

Fig. 10. Principle flowchart of a climate model (black) and necessary extensions for a temperature tagging scheme (red).

\section{Conclusions}

In this study a new methodology is introduced which attributes the influence of individual processes on temperature. This method, called temperature tagging, follows the idea of a fully tagged chemical system (Grewe et al., 2012). Fundamental to this tagging approach is the idea that two quantities are equally important when they are both controlling a process. This allows a decomposition of non-linear forcing terms into individual forcing terms for tagged quantities. The tagged quantities have to be seen as contributions to the total quantity. A cooling process may lead to a negative contribution to surface temperature, leading to a negative tagged temperature, which cannot be regarded as a physical temperature per se, but only as a portion of the total temperature.

A simple climate box model is applied to determine the contribution of atmospheric shortwave ("sw") and longwave absorption to temperature. The longwave absorption is split into three parts: absorption due to non- $\mathrm{CO}_{2}$ greenhouse gases ("nC"); base concentration of $\mathrm{CO}_{2}$ ("bC"); and an enhanced $\mathrm{CO}_{2}$ concentration ("eC"). For each of the four tagging categories, the associated fluxes and the temperature evolution are calculated.

Two experiments were performed. In the first, changes in fluxes and temperatures were investigated starting from 
a situation without an atmosphere (zero absorption) and steadily increasing the absorption to a full atmosphere, which leads to a surface temperature increase from $255 \mathrm{~K}$ to $286 \mathrm{~K}$. This increase in temperature is composed of individual contributions of opposite signs. A decreasing contribution $(-14 \mathrm{~K})$ of the shortwave flux to the surface temperature results from the increasing shortwave absorption of the atmosphere leading to a lower transmittance of radiation to the surface. In the situation of no atmosphere, the surface temperature is caused by the shortwave influx and hence attributed $100 \%$ to the category "sw". The increase in atmospheric absorption leads to a decrease of the contribution of the solar influx to the surface temperature, since the greenhouse effect starts to contribute significantly. The fluxes, which determine the temperatures, are the net shortwave flux of $192 \mathrm{~W} \mathrm{~m}^{-2}$ and the longwave downward flux of $198 \mathrm{~W} \mathrm{~m}^{-2}$, leading to a total of $381 \mathrm{~W} \mathrm{~m}^{-2}$, which equals $286 \mathrm{~K}$ surface temperature. The longwave downward flux of $198 \mathrm{~W} \mathrm{~m}^{-2}$ has a contribution from the emitted radiation from the surface attributed to solar heating (category "sw") of $98 \mathrm{~W} \mathrm{~m}^{-2}$ and $91 \mathrm{Wm}^{-2}$ attributed to the absorption of greenhouse gases. Hence roughly $25 \%\left(91 \mathrm{~W} \mathrm{~m}^{-2}\right.$ out of $381 \mathrm{~W} \mathrm{~m}^{-2}$ ) or $68 \mathrm{~K}$ are attributed to the greenhouse effect.

Therefore, the difference in surface temperature between a situation with and without atmosphere of $31 \mathrm{~K}$ is only a part of the total greenhouse effect of $68 \mathrm{~K}$, if we define the surface temperature caused by longwave absorption as a quantification of the greenhouse effect.

In a second experiment, the $\mathrm{CO}_{2}$ concentration is doubled, which leads to an increase in surface temperature of $1.2 \mathrm{~K}$, resulting from a temperature increase due to $\mathrm{CO}_{2}$ of $1.9 \mathrm{~K}$, due to non- $\mathrm{CO}_{2}$ greenhouse gases of $0.6 \mathrm{~K}$ and a cooling of $1.3 \mathrm{~K}$ due to a reduced importance of the solar heating for the surface and atmospheric temperatures.

To summarise, temperature tagging is an important addition to understanding calculated temperature changes. It allows attributing causes to temperature changes, e.g. temperature changes caused by changes in greenhouse gas emissions and hence enables a deeper interpretation of calculated total changes in temperatures. This study shows that tagging of temperature is possible, i.e. that it is possible to keep track of changes in temperatures during a climate simulation. Including a full temperature tagging into a comprehensive climate model still needs more considerations, but its potential is clearly shown in this study.

Acknowledgements. This work was performed during a sabbatical (DLR-Forschungssemester) at the National Center of Atmospheric Research (NCAR) in Boulder, CO, and I am grateful for them giving me this opportunity. I like to thank J.-F. Lamarque and A. Conley for helpful comments and people at NCAR for their hospitality and H. Garny for helpful discussions.

\section{References}

Andrews, D. G.: An Introduction to Atmospheric Physics, 2nd Edn., Cambridge University Press, New York, 2010.

Barry, R. G. and Chorley, R. J.: Atmosphere, Weather and Climate, 8th Edn., Routledge, New York, USA, 2003.

Bowers, S. A. and Hanks, R. J.: Specific heat capacity of soils and minerals as determined with a radiation calorimeter, Soil Science, 94, 392-396, 1962.

Dole, R., Hoerling, M., Perlwitz, J., Eischeid, J., Pegion, P., Zhang, T., Quan, X. W., Xu, T., and Murray, D.: Was there a basis for anticipating the 2010 Russian heat wave?, Geophys. Res. Lett., 38, L06702, doi:10.1029/2010GL046582, 2011.

Gregory, J. M., Ingram, W. J., Palmer, M. A., Jones, G. S., Stott, P. A., Thorpe, R. B., Lowe, J. A., Johns, T. C., and Williams, K. D.: A new method for diagnosing radiative forcing and climate sensitivity, Geophys. Res. Lett., 31, L03205, doi:10.1029/2003GL018747, 2004.

Grewe, V.: A generalized tagging method, Geosci. Model Dev., 6, 247-253, doi:10.5194/gmd-6-247-2013, 2013.

Grewe, V., Tsati, E., and Hoor, P.: On the attribution of contributions of atmospheric trace gases to emissions in atmospheric model applications, Geosci. Model Dev., 3, 487-499, doi:10.5194/gmd3-487-2010, 2010.

Grewe, V., Dahlmann, K., Matthes, S., and Steinbrecht, W.: Attributing ozone to $\mathrm{NO}_{\mathrm{x}}$ emissions: implications for climate mitigation measures, Atmos. Environ., 59, 102-107, 2012.

Hansen, J., Sato, M., and Ruedy, R.: PNAS plus: perception of climate change, P. Natl. Acad. Sci., 109, E2415-E2423, doi:10.1073/pnas.1205276109, 2012.

Hasselmann, K., Sausen, R., Maier-Reimer, E., and Voss, R.: On the Cold Start Problem in Transient Simulations with Coupled Atmosphere-Ocean Models, Clim. Dynam., 9, 53-61, 1993.

Hilsenrath, J., Beckett, C. W., Benedict, W. S., Fano, W. S., Hoge, L., Masi, J. F., Nuttall, R. L., Touloukian, Y. S., and Wodley, H. W.: Tables of Thermal Properties of Gases, NBS Circular No. 564, 1955.

IPCC: Climate Change 2007: The Physical Science Basis. Contribution of Working Group I to the Fourth Assessment Report of the Intergovernmental Panel on Climate Change, edited by: Solomon, S., Qin, D., Manning, M., Chen, Z., Marquis, M., Averyt, K. B., Tignor, M., and Miller, H. L., Cambridge University Press, Cambridge, UK and New York, NY, USA, 996 pp., 2007.

Jacobsen, M. Z.: Atmospheric Pollution: History, Science and Regulation, Cambridge University Press, UK, 2002.

Kiehl, J. T. and Trenberth, K. E.: Earth's Annual Global Mean Energy Budget, Bull. Am. Meteorol. Soc., 78, 197-208, 1997.

Osborne, F., Stimson, H. S., and Ginnings, D. C.: Thermal properties of saturated water and steam, Bur. Stundards J. Research, 23, 197, in: Handbook of Chemistry and Physics, 53rd Edn., Cleveland, Ohio, D128, (1972-1973), 1939.

Ramanathan, V.: Trace-gas greenhouse effect and global warming: Underlying principles and outstanding issues, Ambio, 27, 187197, 1998.

Smil, V.: The Earth's Biosphere: Evolution, Dynamics, and Change, MIT-Press, USA, 2003.

Edited by: H. Tost 